\title{
A Comprehensive \& Systematic Study of Coincidence Time Resolution and Light Yield Using Scintillators of Different Size and Wrapping
}

\author{
E. Auffray, Member, IEEE, B. Frisch, Member, IEEE, F. Geraci, A. Ghezzi, S. Gundacker, \\ H. Hillemanns, Member, IEEE, P. Jarron, Member, IEEE, T. Meyer, M. Paganoni, K. Pauwels, M. Pizzichemi, and \\ P. Lecoq, Member, IEEE
}

\begin{abstract}
Over the last years, interest in using time-of-flightbased Positron Emission Tomography (TOF-PET) systems has significantly increased. High time resolution in such PET systems is a powerful tool to improve signal to noise ratio and therefore to allow smaller exposure rates for patients as well as faster image acquisition. Improvement in coincidence time resolution (CTR) in PET systems to the level of 200 ps $F W H M$ requires the optimization of all parameters in the photon detection chain influencing the time resolution: crystal, photodetector and readout electronics. After reviewing the factors affecting the time resolution of scintillators, we will present in this paper the light yield and CTR obtained for different scintillator types (LSO:Ce, LYSO:Ce, LGSO:Ce, LSO:Ce:0.4Ca, LuAG:Ce, LuAG:Pr) with different cross-sections, lengths and reflectors. Whereas light yield measurements were made with a classical PMT, all CTR tests were performed with Hamamatsu-MPPCs S10931-050P. The CTR measurements were based on the time-over-threshold method in a coincidence setup using the ultra fast amplifier-discriminator chip NINO and a fast oscilloscope. Strong correlations between light yield and CTR were found. Excellent results have been obtained for LYSO crystals of $2 \times 2 \times 10 \mathrm{~mm}^{3}$ and LYSO pixels of $0.75 \times 0.75 \times$ $10 \mathrm{~mm}^{3}$ with a CTR of 175 ps and 188 ps FWHM, respectively.
\end{abstract}

Index Terms-Lutetium-aluminum garnets, lutetium-oxy-orthosilicate (LSO), multi-pixel photon counters (MPPCs), silicon photomultipliers (SiPM), time-based readout, time-over-threshold discrimination.

\section{INTRODUCTION}

$\mathbf{T}$ IME of flight in PET has become, as a result of the emergence of fast silicon photomultipliers (SiPMs), an increasingly attractive instrument to enhance the quality of medical imaging with far reaching impacts on patient care and hospital expenditures. Furthermore, certain diagnostic methods such as novel endoscopic interventions employing PET in addition to

Manuscript received October 24, 2012; revised February 28, 2013; accepted May 07, 2013. Date of publication July 23, 2013; date of current version October 09,2013. The research leading to these results received support from the European Union 7th Framework Program (FP7/2007-2013) under Grant Agreements 256984 (EndoTOFPET-US) and 241851 (ENVISION). This work was carried out in the frame of the Crystal Clear Collaboration.

E. Auffray, B. Frisch, S. Gundacker, H. Hillemanns, T. Meyer, K. Pauwels, and P. Lecoq are with the European Organization for Nuclear Research (CERN), 1211 Geneva 23, Switzerland (e-mail: etiennette.auffray@cern.ch).

F. Geraci, A. Ghezzi, M. Paganoni, and M. Pizzichemi are with the University of Milano-Bicocca, 20126 Milano, Italy.

P. Jarron is with INFN Torino, 10125 Torino, Italy.

Color versions of one or more of the figures in this paper are available online at http://ieeexplore.ieee.org.

Digital Object Identifier 10.1109/TNS.2013.2270089 conventional modes may fail if not a tight time constraint is used in PET to reduce statistical noise and suppress highly uncorrelated background events that prevent an efficient and reliable tumor image reconstruction. Such background, for example, is provoked by annihilation photons originating from marker take-up in neighboring organs other than the target organ under investigation [1]-[7]. Recognizing the necessity of TOF in such applications implies a rigorous search for the highest possible coincidence time resolution (CTR). A figure of merit for such a resolution should be 200 ps FWHM or better, which corresponds to a spatial resolution along the LOR of $\leq 30 \mathrm{~mm}$.

An important premise for this study is to focus on realistic instrumentation and detector components that are to be used in future TOF-PET applications, with the aim not to compromise detector-efficiency in a search for highest time resolution. In other words, while it is known that, e.g., shorter crystals yield faster time response, the advantage of higher time resolution is easily traded off against a significant loss of detector efficiency. This is why our investigations concentrate on crystals with $\mathrm{L} \geq 8 \mathrm{~mm}^{1}$. Similarly, on grounds of complexity and cost, scintillators made of $\mathrm{LaBr}_{3}$ are not part of this study.

Our system is made of scintillators typically of $2 \times 2 \times 10$ $\mathrm{mm}^{3}$ or, as a prerequisite for adequate spatial resolution in the endoscopic PET-probes for EndoTOFPET-US [1], by pixels with dimensions of $0.75 \times 0.75 \times 10 \mathrm{~mm}^{3}$, together with a $\mathrm{SiPM}$ as photosensitive detector, and its associated electronics. The challenge to achieve the highest possible time resolution can only be met if every component of the detection system is optimized in terms of light production, light transport, optical coupling, photo-conversion and noise. In a previous study [8] we had addressed this question in terms of a systematic search for optimum SiPM performance, whereas this study centers on an understanding of scintillator behavior in light of their timing performance under differing physical (and chemical) conditions such as scintillator type, crystal or pixel size and reflector material. Since the focus is on scintillators we will only give a brief description of the photodetectors and their electronic circuitry used in the light-yield (LY) and CTR test benches. LY measurements are typically made with standard PMTs and with the crystals under investigation mounted "vertically", i.e., small face on, and coupling grease at the interface. CTR measurements throughout make use of two SiPMs or MPPCs

\footnotetext{
${ }^{1}$ Some crystals with $\mathrm{L}<5 \mathrm{~mm}$ were included so as to investigate time resolution and light yield as a function of crystal length.
} 
by Hamamatsu that are coupled differentially to a pair of fast amplifier-discriminators directly read out by a high bandwidth (3.5 GHz) oscilloscope. More explicit descriptions on the test setups are found in Section III and are also given in [9] and [10] for the discriminator-amplifier NINO, and [11] for the high bandwidth oscilloscope used.

\section{Principal Influences ON Photon Timing AND LIGHT YIELD}

With reference to previous investigations [12]-[15] we will, at this point and with special emphasis on crystals, give a short review of the principal effects limiting the time resolution in a TOF-PET setup, as well as the dominant factors affecting the LY of scintillating crystals used in such a setup. In the ensuing studies we will show that total light output and time resolution are strongly linked and that both should be optimized together [16]-[18].

\section{A. Photostatistics}

The time resolution achievable with slowly decaying scintillators is limited primarily by the statistics of photon production converted into electrons in a detector [19], [20]. Poisson statistics dictate that the most probable time interval $t_{\mathrm{Q}}$ of detecting the Qth converted photon derives from the probability density of detecting each of the photoelectrons $\mathrm{R}$ produced per excitation, and from their exponentially decaying emission rate. Thus, the intrinsic time resolution, imposed by this effect alone [20], [21], is derived from two parameters only: the scintillator decay time and the total photoelectron yield R [12]:

$$
t_{Q}=\tau_{f} \cdot \ln \left[\frac{1}{2}\left(Q-R+\sqrt{(Q-R)^{2}+4 R}\right)\right]
$$

$\tau_{\mathrm{f}}$ denotes the decay or fall time of the photonic emission rate and $\mathrm{R}$ the total number of photoelectrons produced.

From (1) we learn that optimum time resolution $\left(\mathrm{t}_{\mathrm{Q}}=\mathrm{min}\right)$ is achieved if both $\tau$ and $\mathrm{Q}$ are minimum and $\mathrm{R}$ is large. $\mathrm{R}$ and $\tau$ are typical scintillator characteristics whereas the goal to reach $\mathrm{Q}=1$ is also determined by the photodetector gain and -noise plus the electronics-discriminator threshold.

It was, however, suggested by Y. Shao in 2007 [22] that for fast scintillators, such as e.g., LSO, LuAG:Pr, LuYAP and $\mathrm{LaBr}_{3}: \mathrm{Ce}$, the above formalism no longer represents a valid description of the experimental results failing to take into account the non-zero rise time of the crystal luminescence that leads to the scintillation process. Therefore a bi-exponential timing expression for the light emission that comprises both the scintillation rise- and fall time needs to be applied which, following integration, describes the number of photoelectrons hitting the photodetector in the time interval between 0 and $t$ :

$$
Q(t)=R \frac{\tau_{r}+\tau_{f}}{\tau_{f}^{2}} \int_{0}^{t} e^{-\frac{t}{\tau_{f}}}\left(1-e^{-\frac{t}{\tau_{r}}}\right) d t
$$

where $\tau_{\mathrm{r}}$ and $\tau_{\mathrm{f}}$ denote the scintillation rise- and fall time, respectively, and R (as before) the total photoelectron yield per excitation. Assuming $\tau_{\mathrm{r}} \ll \tau_{\mathrm{f}}$ and expanding the exponentials
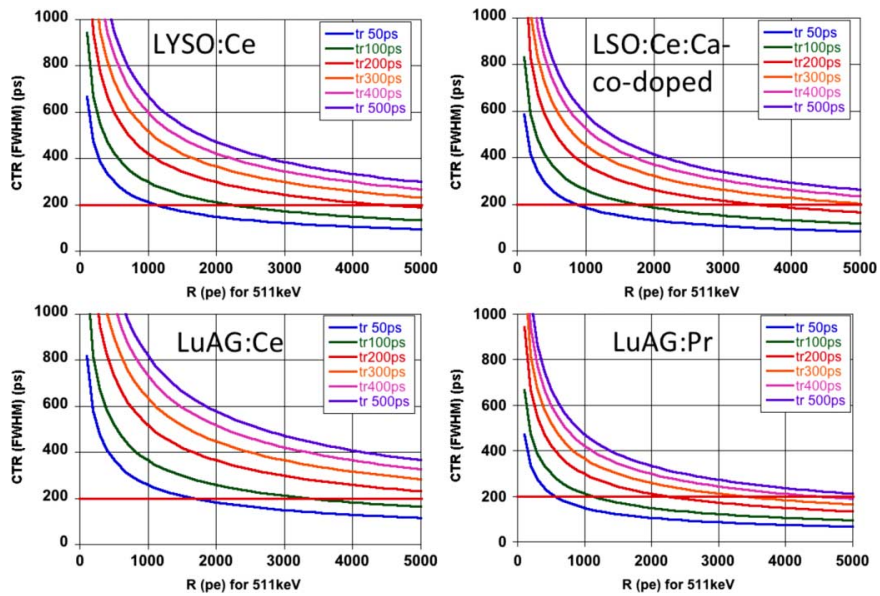

Fig. 1. Estimated coincidence time resolution for four scintillation crystals with different light decay times as a function of photoelectron yield for five different scintillation rise times. Note, no other effects influencing the CTR are included.

in (2) to second order, leads to an approximate expression for $\mathrm{Q}(\mathrm{t})$ :

$$
Q(t) \approx \frac{R}{2} \frac{t^{2}}{\tau_{r} \tau_{f}}
$$

from which we derive the approximate average arrival time $\mathrm{t}_{\mathrm{Q} 1}(\sigma)$ of the first photon as:

$$
\left\langle t_{Q_{1}}\right\rangle \approx \sqrt{\frac{2}{R} \cdot \tau_{r} \tau_{f}}
$$

On grounds of Poissonian statistics alone and neglecting single-photon time resolution, this expression denotes the expected latency (mean) of the Qth photon and consequently the uncertainty (or standard deviation) on the arrival time of the photon.

Expressed in terms of coincidence time resolution (CTR) and FWHM, relation (4) then becomes:

$$
\mathrm{CTR}_{Q_{1}} \approx \sqrt{\frac{2}{R} \cdot \tau_{r} \tau_{f}} *(2.36 \cdot \sqrt{2})
$$

It must be noted, however, that in the context of overall timing resolution this approximation is a simplification as, e.g., SiPM transit time spread, being typically of order hundred picoseconds, is totally neglected in this picture [23], [24]. The aim of this paper, however, is to concentrate on scintillators and ascertain their trends in CTR irrespective of other instrumental influences, albeit shown in a separate work by Gundacker et al. [8]. Hence, Fig. 1, according to (5), merely exhibits the "characteristic" CTR of four different scintillator types, each with its specific light decay time constant $\tau_{\mathrm{f}}$, as a function of total photoelectron yield $\mathrm{R}$ and parameterized for five different scintillation rise times $\tau_{\mathrm{r}}$ ranging from 50 to $500 \mathrm{ps}$ [25]. Even if (the above mentioned) photodetector timing uncertainties were to be (quadratically) included, the arbitrarily chosen CTR limit of 200 ps FWHM would not change dramatically. 
TABLE I

Minimally ReQuired Photoelectron Yield, R, to SATISFy $\leq 200$ PS CRT

\begin{tabular}{|c|c|c|c|c|c|}
\hline \multirow{2}{*}{ Crystal } & \multirow{2}{*}{$\begin{array}{c}\text { Decay } \\
\text { Time" } \\
{[n s]} \\
\end{array}$} & \multicolumn{4}{|c|}{ Rise Time $\tau_{\bullet}[\mathrm{ps}]$} \\
\hline & & 50 & 100 & 200 & 500 \\
\hline LYSO:Ce & 40 & $1 ’ 060$ & $2 ’ 120$ & 4'230 & $10^{\prime} 580$ \\
\hline $\mathrm{LSO}: 0.4 \mathrm{Ca}$ & 31 & 820 & $1 ' 640$ & 3’280 & $8 ' 200$ \\
\hline LuAG:Ce & 60 & 1'590 & 3'180 & $6 ’ 350$ & $15 ’ 870$ \\
\hline LuAG:Pr & 20 & 530 & $1 ’ 060$ & $2 ’ 120$ & 5'290 \\
\hline
\end{tabular}

*) Note, these are average values. For rise time measurements see [25].

Under the assumption that a minimum CTR of $\sim 200 \mathrm{ps}$ FWHM is to be attained, these simplified calculations allow us to extract a framework in which these crystals must be operated and, hence, a priori exclude unsuitable scintillators on the basis of inappropriate light yield and/or too long an emission decay time. As an example, Table I shows for the four shown crystal types of what value the minimum number of photoelectrons $\mathrm{R}$ must be in order to satisfy a minimum CTR of $\leq 200$ ps FWHM.

Taking the example of LuAG:Ce, one notices that, for a signal rise time of $200 \mathrm{ps}, \gtrsim 6000$ photoelectrons (p.e.) are required to obtain a CTR of 200 ps, whereas experimentally a p.e. yield R of only 2000 was measured. Hence, due to its long decay time constant of $60 \mathrm{~ns}, \mathrm{LuAG}$ :Ce will not reach a time resolution of 200 ps.

Another criterion for a scintillator to prove suitable in this respect is its characteristic emission wavelength, for most (if not all) $\mathrm{SiPMs}^{2}$ exhibit their peak sensitivity in the blue (400 $\mathrm{nm}$ ) region. Thus, despite its comparably short decay time constant, also LuAG:Pr, because of its unfavorable emission in the ultra-violet regime of 320-370 $\mathrm{nm}$ and hence low p.e. yield, is likely to be dismissed from the list of TOF-suitable scintillating crystals. The LSO family seems to be more adequate, and therefore we performed systematic LY and CTR studies on $\mathrm{L}(\mathrm{Y} / \mathrm{G}) \mathrm{SO}$ crystal samples from various producers. Time resolution measurements are thus supplemented by analog LY measurements searching for correlations of timing performance and LY.

\section{B. Geometric Effects}

This chapter shortly reviews light propagation in the scintillator with focus on the scintillator's size or length. Besides the large improvements in scintillators' characteristic light emission rate, a dominant factor on travel time and latency is the length of the crystal [26]. In the (2-D) calculations of [12] we find that only a fraction of the light, emitted uniformly from the gamma conversion point, will be detected by the photodetector. Constrained by the different indices of refraction at the crystal's interfaces (air and silicon grease) only light emitted within well defined angular regions can reach the photodetector. This can be light that is emitted directly in the forward direction towards the photodetector but also photons travelling backwards and undergoing multiple reflections at the crystal boundaries before exiting the crystal into the photodetector. These 'cones' of light, which are limited by the critical angle of reflection, are shown in

\footnotetext{
${ }^{2}$ This does not apply to photocathode-based sensors such as PMTs and MCPs.
}

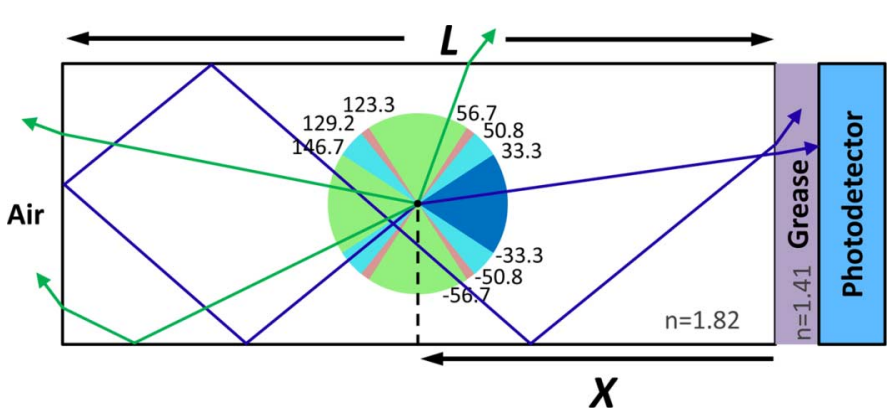

Fig. 2. Illustration of the geometry of light emission and the fraction of light reaching the photodetector. Only $\sim 30 \%$ of the produced light emitted within light and dark blue cones will eventually enter the photodetector whereas, in the absence of wrapping, light from the green cones escapes from the crystal. At the critical angles for the crystal-grease interface of $50.8^{\circ}$ and $129.2^{\circ}$ (pink zone) no light will ever exit the crystal.

blue (light and dark) in Fig. 2. It should be noted, however, that these calculations do not take into account the effect of reflective material wrapped around the crystal. These external reflectors backscatter light into the crystal and hence eventually into the photodetector. This light (within the green cones) would otherwise be lost. On the other hand, light emitted from within the pink zones confined by the critical reflection angle due to the crystal-grease interface will never exit the crystal.

Therefore, assuming gamma conversion at position $\mathrm{x}$ along the crystal axis inside a crystal of length L, two angular zones that satisfy light collection at the photodetector are singled out:

1) Forward direction:

$-50.8^{\circ} \leq \theta \leq 50.8^{\circ}$ where the propagation time is given by:

$$
t_{\text {prop }}=\frac{n}{c} \cdot \frac{x}{\cos (\theta)}
$$

or averaged over the crystal length L:

$$
\left\langle t_{\text {prop }}\right\rangle=\frac{n}{2 c} \cdot \frac{L}{\cos (\theta)}
$$

2) Backward direction (two allowed zones): $129.2 \leq \theta \leq 146.7$ and $-129.2 \leq \theta \leq-146.7$, with

$$
t_{\text {prop }}=\frac{n}{c} \cdot \frac{2 L-x}{\cos (\theta)}
$$

or averaged over the crystal length L:

$$
\left\langle t_{\text {prop }}\right\rangle=\frac{n}{2 c} \cdot \frac{3 L}{\cos (\theta)}
$$

Therefore, taking into consideration the difference between direct (forward) and longest backward photon paths, the maximum contribution of light travel to the coincidence time resolution, over the crystal length ${ }^{3}$, is $57 \mathrm{ps}$ (for $\mathrm{L}=5 \mathrm{~mm}$ ),

\footnotetext{
${ }^{3}$ Note, this is not strictly correct, as our simplified model does not take into account the gamma absorption length in the crystal, which is non-uniform along the crystal axis. Thus, the flight time of the gamma from the crystal entry- (L) to the conversion point $(\mathrm{x})$ is not taken into account.
} 
$114 \mathrm{ps}(\mathrm{L}=10 \mathrm{~mm})$ reaching $230 \mathrm{ps}$ for $\mathrm{L}=20 \mathrm{~mm}$ of crystal length. These values, however, if spread statistically in a Gaussian fashion, should then be properly 'weighted' so as to derive an average timing uncertainty owing to photon travel inside the crystal. Assuming the minimum and maximum photon path lengths to be within $6 \sigma$ of a Gaussian, the above values would reduce to an average value with FWHM of $22 \mathrm{ps}, 45 \mathrm{ps}$ and $90 \mathrm{ps}$, respectively. It should be noted that the above calculations are in 2-dimensions only and therefore represent only an approximation to a more rigorous approach if made in 3-D.

From these assumptions we deduce that in current PET facilities, where crystals of $\sim 20 \mathrm{~mm}$ are used, the contribution of light travel to the overall system time resolution is non-negligible. Therefore, in an aim to optimize both efficiency and time resolution, our figure of merit for minimum crystal length is 10 $\mathrm{mm}$.

\section{Surface Effects and Wrapping of Crystals}

Comparative studies by M. Kronberger et al. [27], [28] of simulations and experimental results of light yield in crystals have shown good agreement for bare and polished (Fresneltype) crystals. However, notwithstanding a missing conclusive simulation of photon transport in crystals that includes various types of reflective material, the wrapping of crystals with reflectors, in particular with Teflon, has a pronounced beneficial effect on light output [29], [30] and, despite the increased number of surface reflections, also on timing performance. The paper will address this question in more detail and experimentally study the influence of wrapping in terms of reflector material, surfaces to be covered (top and/or lateral crystal faces).

\section{EXPERIMENTAL SETUPS}

Two setups were used in parallel. One test bench was dedicated to the light yield measurements and another to the study of the coincidence time resolution. Light yield measurements were made with a PMT (Photonis XP2020Q) as photodetector. SiPMs are not suitable for this type of measurement because saturation effects set in at high light input owing to the limited number of individual photosensitive pixels (SPADs).

The coincidence circuit for the timing measurements, however, does make use of SiPMs and is shown in Fig. 3. Only one type of SiPM was used in two identical back-to-back detector arms, i.e., the Hamamatsu-MPPC S10931-050P, with a SPAD size of $50 \mu \mathrm{m}$. From an earlier study [8] it was found to be the fastest for these purposes. The operating bias of this SiPM, optimized for highest time resolution, was $72.4 \mathrm{~V}, 2 \mathrm{~V}$ above the measured breakdown voltage.

To reject ground- and supply voltage noise the SiPMs are connected differentially [10] to the front-end amplifier-discriminator chip NINO. In such a scheme both the anode and cathode of the SiPM feed the avalanche signal to the amplifier with the advantage of effectively suppressing common mode noise picked up in the signal path.

The NINO discriminator employs the time-over-threshold technique. It produces a square pulse with a width proportional to the charge of the input signal above the discriminator threshold. This technique, together with the fast timing characteristics of the chip (time jitter $\leq 25$ ps r.m.s.), allows deriving

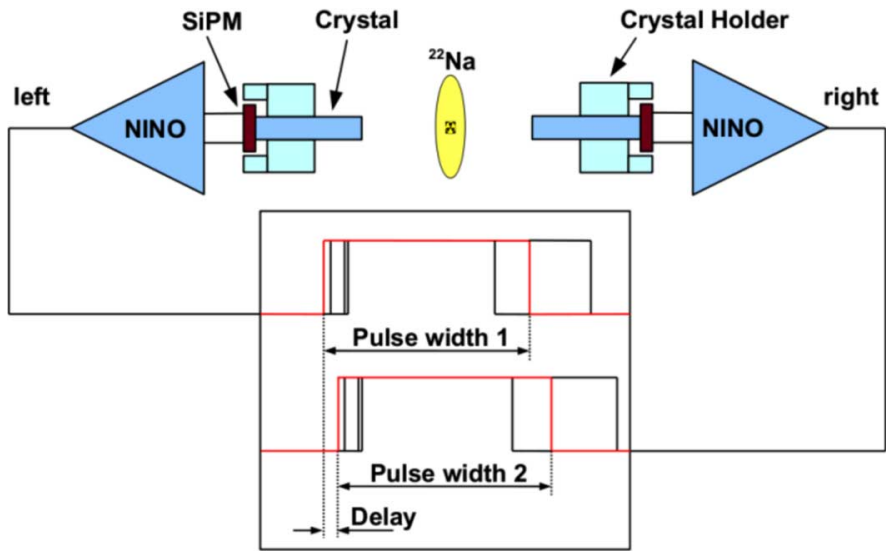

Fig. 3. Schematic of the test setup for the coincidence time measurements. The SiPM signals are fed into the CERN-developed NINO chip, an ultra fast discriminator-amplifier. A high-bandwidth oscilloscope, LeCroy DDA 735Zi (40 GSamples/s), records the dual pulse widths from the photodetectors plus their mutual delay in the time-based-readout scheme.

from the pulse width the energy information and from the leading edge the precise time stamp ${ }^{4}$. The NINO output thus encodes both energy and time information in one digital pulse.

A high bandwidth oscilloscope, LeCroy DDA 735Zi (40 GS/s), recorded the dual pulse widths from the two NINO outputs plus their mutual delay. The logic of the scope is set such that only coincident events are collected irrespective of their energy; i.e., events with the full energy from the photoelectric effect and Compton scattered events. The scope records these events in lists or histograms keeping track of the mutual time delay between coincident photons and the energy of the photons. The energy tag of the photons is needed to eliminate (offline) non-photoelectric or Compton events for the refinement of the coincidence time spectrum.

The crystals were polished (specular) on all six faces and coupled to the photodetector with optical grease.

All measurements were performed at room temperature $\left(20^{\circ} \pm 0.1^{\circ} \mathrm{C}\right)$ in a thermally controlled dark box.

\section{TEST PROCEDURES}

\section{A. Light Yield Measurements}

All measurements were made with the scintillating crystal wrapped with Teflon and mounted "vertically", i.e., with its small square face on the PMT window. For all tests optical grease ("Pate 7", Rhodorsil silicon compound with $\mathrm{n}=1.41$ ) was applied to the crystal/PMT interface. ${ }^{137} \mathrm{Cs}$-gammas served as the energy standard for the spectroscopic measurements. Fits of the photoelectric peak normalized to the single-photoelectron response of the PMT allowed to derive the light yield commonly expressed in terms of $\mathrm{Ph} / \mathrm{MeV}$. An example of such an energy spectrum, made with this source, is shown in Fig. 4.

\section{B. Timing Measurements}

Reaching the best timing performance with commercially available and low cost crystals, operated together with SiPMs,

\footnotetext{
${ }^{4}$ The ensemble of the Hamamatsu SiPM (MPPC S10931-050P) and NINO allowed triggering at the one-photon threshold.
} 


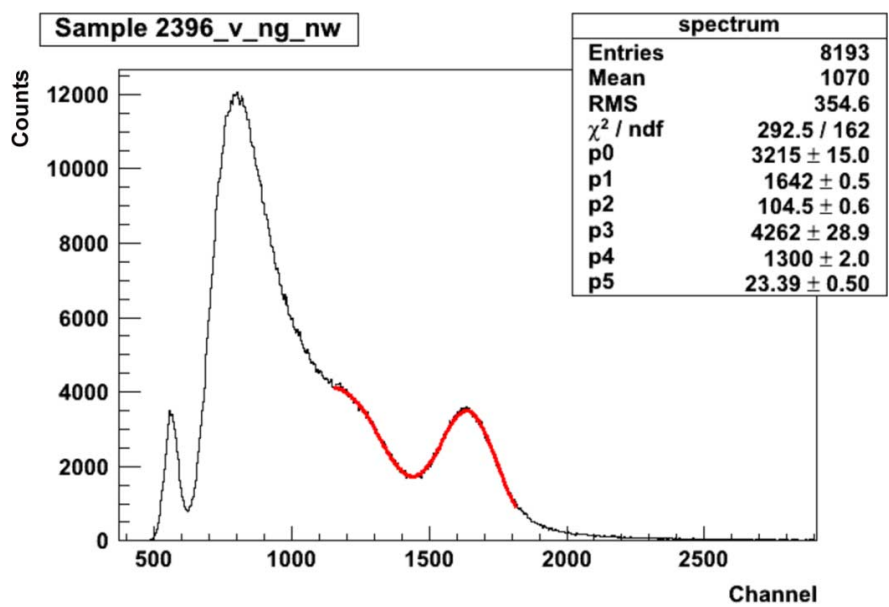

Fig. 4. ${ }^{137} \mathrm{Cs}$ spectrum made with an AGILE LSO:Ce:Ca-co-doped crystal of $2 \times 2 \times 20 \mathrm{~mm}^{3}$ size. The position of the photoelectric peak w.r.t. the single photoelectron response of the PMT determines the $\mathrm{LY}(\mathrm{Ph} / \mathrm{MeV})$. Note, the photodetection efficiency (PDE) of the used PMT (Photonis XP2020Q) was prior determined from the crystal's emission spectrum convoluted with the measured PMT's QE. PDE in this measurement is $22 \%$.

is the ultimate goal of this study. Coincidence measurements with back-to-back $\mathrm{e}^{+} \mathrm{e}^{-}$-annihilation photons provide the best test bench for this purpose. This has been described in detail in [8] and [14]. Depending on the specific study underway, the crystals were wrapped with reflectors - in most cases with Teflon - and also mounted with their small face onto the SiPM using silicon grease at the interface. The ensemble of the SiPM and the NINO amplifier-discriminator, both operated in differential mode as described in Section III, has proven to yield excellent timing accuracy with no need for additional signal processing.

At this point we note that tests were repeated several times so as to eliminate systematic errors from improper mounting of the crystals, and bias fluctuations, and accidental effects. To "gauge" the timing quality of the studied crystals the delay time spectrum was recorded for each pair of same crystals, which was then refined through the selection of photoelectric events. The resulting coincidence time spectrum and its Gaussian fit-see Fig. 5 as an example-will then be tabulated according to the criteria under study.

\section{RESUlTS}

\section{A. Comparison of Two Different Crystal Compounds Based on Lutetium}

This study investigated two different classes of lutetiumbased crystals:

a) The commonly used lutetium ortho-silicates such as $\mathrm{Lu}_{2} \mathrm{SiO}_{5}$ (LSO) and its derivatives such as LYSO, or LGSO. In this species, some of the lutetium atoms are replaced by yttrium or gadolinium, respectively. These scintillators are dense $\left(\leq 7.4 \mathrm{~g} / \mathrm{cm}^{3}\right)$ and if doped with cerium characterized by a decay time of approximately $40 \mathrm{~ns}$ and a fairly high light output of $\geq 20^{\prime} 000 \mathrm{Ph} / \mathrm{MeV}$; New are so-called calcium-co-doped LSO crystals that were also investigated by us in view of their substantially

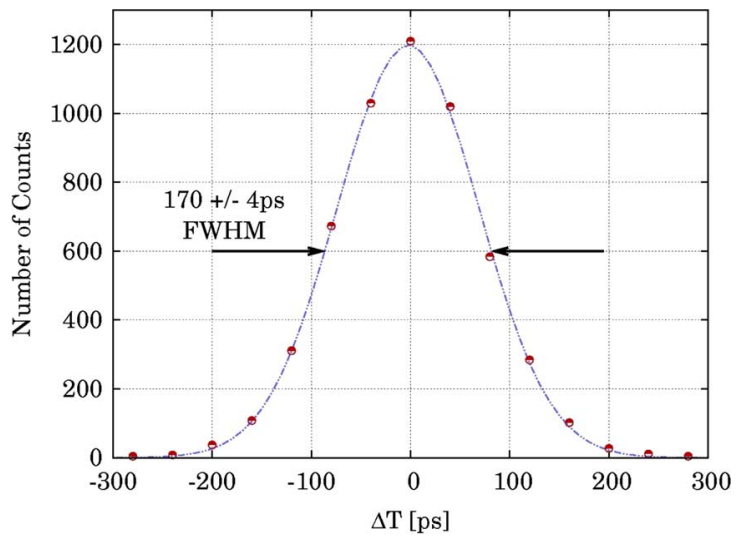

Fig. 5. Example of a delay time spectrum taken with two crystals (LSO:Ce: $\mathrm{Ca} / 2 \times 2 \times 10 \mathrm{~mm}^{3}$ ) in the setup above. Coincident back-to-back photons came from a ${ }^{22} \mathrm{Na}$ source. After photopeak selection, the CTR for that pair of crystals was derived. SiPM bias was $72.4 \mathrm{~V}$.

TABLE II

Light Yield AND COINCIDENCE TIME RESOLUTION OF LUAG AND LYSO

\begin{tabular}{lcccc}
\hline \hline Crystal & $\begin{array}{c}\text { Size } \\
{\left[\mathbf{m m}^{3}\right]}\end{array}$ & $\begin{array}{c}\text { Decay Time } \\
{[\mathbf{n s}]}\end{array}$ & $\begin{array}{c}\text { Light Yield } \\
{[\mathbf{k P h} / \mathbf{M e V}]}\end{array}$ & $\begin{array}{c}\text { CTR } \\
{[\mathbf{p s}]}\end{array}$ \\
\hline \hline LuAG:Ce & $2 \times 2 \times 8$ & 60 & 15.0 & $872 \pm 50$ \\
LuAG:Pr & $2 \times 2 \times 8$ & 20 & 10.4 & $672 \pm 30$ \\
LYSO:Ce & $2 \times 2 \times 8$ & 40 & 13.0 & $230 \pm 4$ \\
\hline \hline
\end{tabular}

Note: All crystals are fully polished and wrapped with Teflon. Light yield was measured with the PMT Photonis XP2020Q with a PDE of $7.4 \%$ for LuAG:Ce, $20.5 \%$ for LuAG:Pr, and $22.2 \%$ for LYSO.

reduced emission decay time of $\sim 30$ ns notwithstanding a tolerable loss in light yield [31].

b) The lutetium aluminum garnets, $\mathrm{Lu}_{3} \mathrm{Al}_{5} \mathrm{O}_{12}$ (LuAG). This class of materials can be as dense as $6.73 \mathrm{~g} / \mathrm{cm}^{3}$ and its light emission decay time as low as $\sim 20 \mathrm{~ns}$ if the crystal is doped with praseodymium.

Table II compares the characteristic features of LuAG:Ce, LuAG:Pr and LYSO:Ce, as well as the light yield of the crystals and the coincidence time resolution achieved with a pair of such crystals.

In line with our assumptions made in Section II.A and shown in Table I, LuAG crystals are deemed unsuitable for high time resolution applications, albeit a very short emission decay time of $20 \mathrm{~ns}$ found for LuAG:Pr, and despite a relatively high light yield as in LuAG:Ce. The short emission time of the praseodymium-doped crystal is largely offset by the crystal's emission in the UV where SiPM-photodetectors mostly exhibit poor PDE. The cerium-doped crystal, despite its comparably high light yield, suffers from too long a decay time in its light emission rate.

The following studies then concentrated on LSO-type crystals, produced by various manufacturers.

\section{B. Light Yield and Coincidence Time Resolution in LSO, LYSO, LGSO and LSO:Ce:Ca(0.4\%)-co-Doped}

In this section different LSO-type crystals from various manufacturers have been studied in terms of LY and CTR. Furthermore the emission decay time constant $\tau_{\mathrm{f}}$ was measured with a high bandwidth oscilloscope. These measurements illustrate the 
TABLE III

LSO-Type Crystals From Various Producers AND their Performance

\begin{tabular}{lcccc}
\hline \hline Crystal & $\begin{array}{c}\text { Manu- } \\
\text { facturer }\end{array}$ & $\begin{array}{c}\text { Decay } \\
\text { Time } \\
{[\mathbf{n s}]}\end{array}$ & $\begin{array}{c}\text { Light } \\
\text { Yield } \\
{[\mathbf{k P h} / \mathbf{M e V}]}\end{array}$ & $\begin{array}{c}\text { CTR } \\
\text { [ps] }\end{array}$ \\
\hline \hline LGSO:Ce & Hitachi & 42.50 & 19.50 & 176 \\
LYSO:Ce & SIPAT & 42.60 & 19.00 & 206 \\
LYSO:Ce & Proteus & 41.40 & 18.00 & 175 \\
LYSO:Ce & CPI & 45.00 & 17.40 & 184 \\
LSO:Ce & PML & 47.00 & 16.30 & 206 \\
LSO:Ce & CTI & 43.50 & 23.00 & 190 \\
LSO:Ce:Ca & Agile & 32.00 & 15.70 & 170 \\
\hline \hline
\end{tabular}

Note: All crystals are $2 \times 2 \times 10 \mathrm{~mm}^{3}$ in size, fully polished and wrapped with Teflon. Light yield was measured with a PMT (Photonis XP2020Q and 22\% PDE); CTR with the Hamamatsu MPPC S10931-050P with 50 $\mu$ m SPAD size and $72.4 \mathrm{~V}$ bias optimized for highest time resolution [8]. NINO threshold is at $100 \mathrm{mV}$ equivalent to the one-p.e.-level.

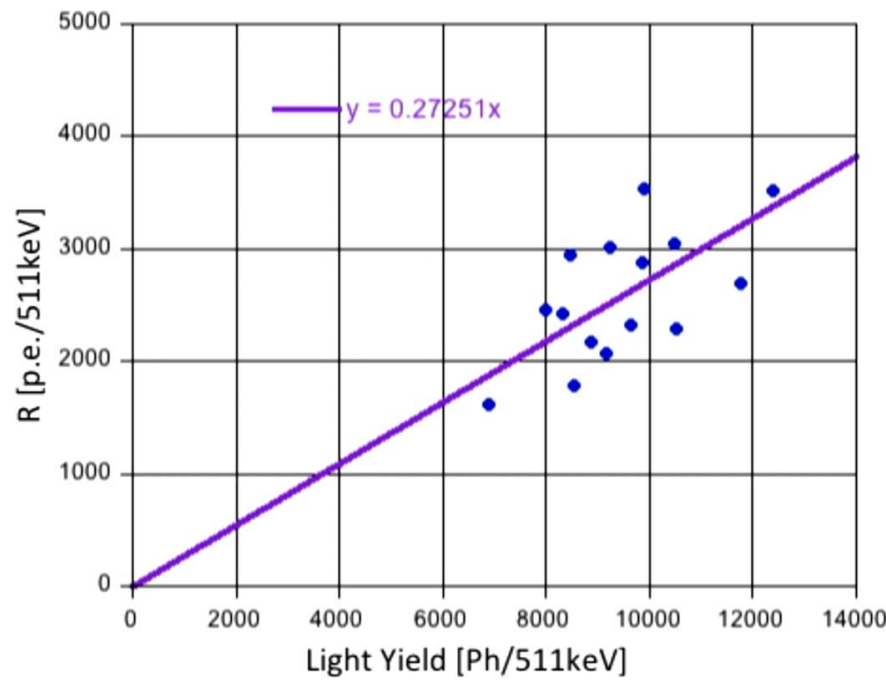

Fig. 6. Correlation of light yield (abscissa) from PMT measurements, and R the detected photoelectron yield (ordinate) from CTR measurements and applying (5) with a rise time of $100 \mathrm{ps}$ and a measured decay time for each sample. Note, the graph intercept was 'forced' to zero. The values are obtained with full crystal wrapping.

slightly different light emission rates among the test samples, even within the same stoichiometric species. These differences are most likely accounted to small variations in doping concentrations and crystal purity, resulting, e.g., from differences in crystal growth. Note all CTR results are within \pm 10 ps unless quoted otherwise.

Table III lists these crystal samples-all made of $2 \times 2 \times 10$ $\mathrm{mm}^{3}$ size - together with the measured LY and CTR.

An important outcome of this investigation is the excellent timing performance of LSO:Ce: $\mathrm{Ca}(0.4 \%)$-co-doped whose short emission decay time outweighs the crystal's lower light yield. Taking the CTR results of LYSO:Ce and LSO:Ce:Ca and "overlaying" them with our estimated (from (5)) CTRs in Fig. 1 (upper two plots), good agreement is seen at a p.e.-yield $\mathrm{R}$ of 2000 and a scintillation rise time of $<100 \mathrm{ps}$. A more detailed comparison of the actually detected photoelectrons and the crystal LY, both measured independently, gives an idea of the overall photodetection efficiency PDE of the system. To

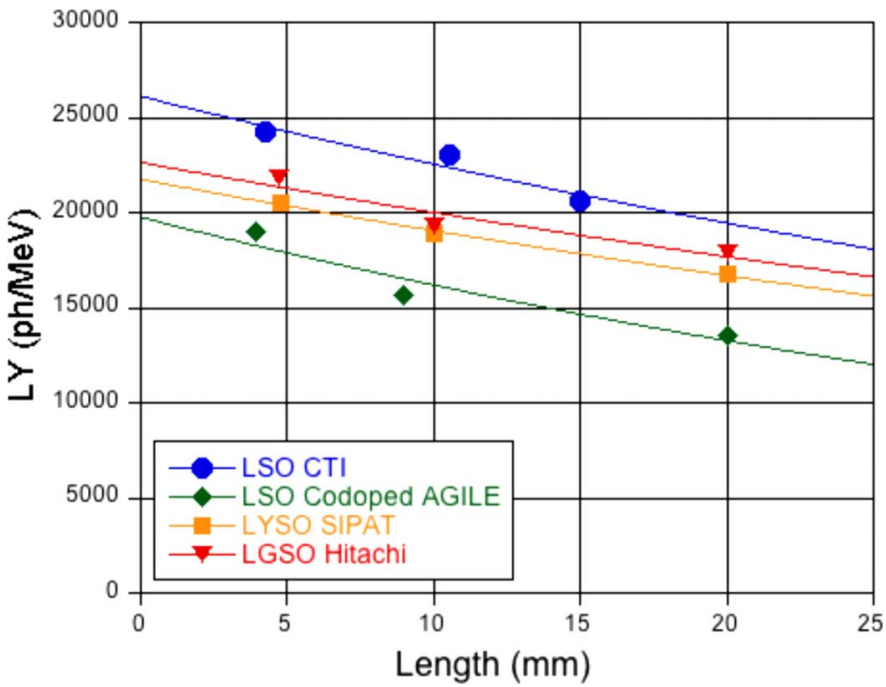

Fig. 7. LY of four different LSO specimen with length of $\sim 5,10,15$ and $20 \mathrm{~mm}$. The values are obtained with full crystal wrapping.

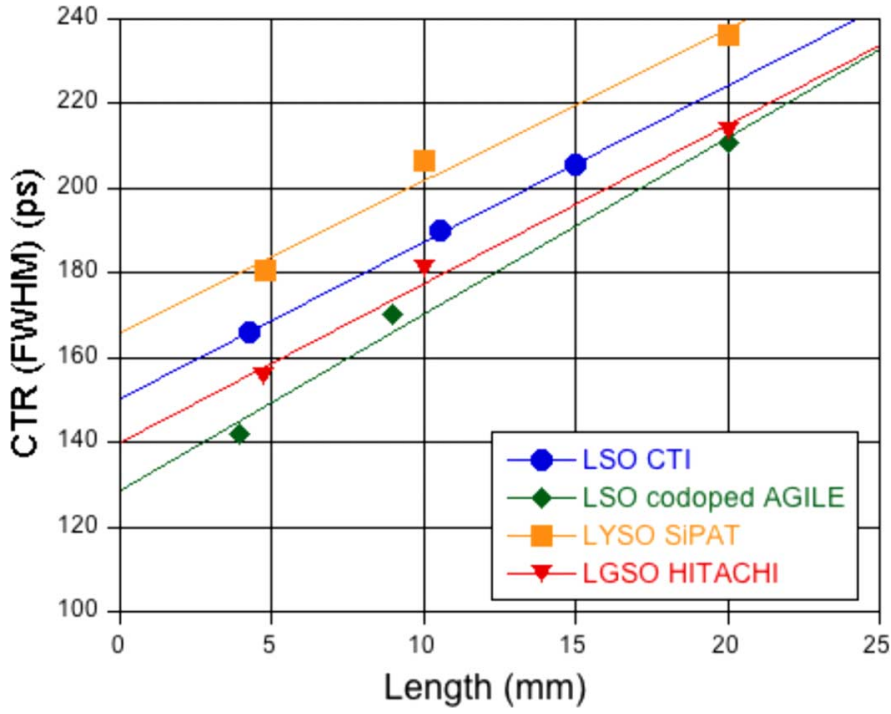

Fig. 8. CTR as a function of crystal length.

illustrate this correlation, while applying (5) for the p.e.-yield R, Fig. 6 shows the relationship between LY and R for all LSO-type crystals, irrespective of their length and manufacturer.

The general behavior of the data shows that an average PDE of $\sim 27 \%$ can be deduced, in fair agreement with the measured value of $\sim 30 \%$ in [32].

\section{Light Yield and Coincidence Time Resolution in LSO-Type Crystals as a Function of Crystal Length}

The following study describes the behavior of LY and CTR as a function of crystal length. In some cases 'long' $(20 \mathrm{~mm})$ crystals were successively cut to $\sim 10 \mathrm{~mm}$ and $\sim 5 \mathrm{~mm}$ length, re-polished and re-wrapped for consecutive measurements. Fig. 7 shows the behavior of LY with crystal length, and Fig. 8 that of the CTR.

Within the limited measured range, light loss is roughly between 10\%/cm (LGSO) and 26\%/cm (LSO:Ce:Ca-co-doped), 
TABLE IV

LY AND CTR IN CRYSTALS OF DifFERENT CROSS SECTIONS

\begin{tabular}{|c|c|c|c|c|}
\hline \multirow{2}{*}{ Crystal } & \multicolumn{2}{|c|}{$2 \times 2 \times 10 \mathrm{~mm}^{3}$} & \multicolumn{2}{|c|}{$0.75 \times 0.75 \times 10 \mathrm{~mm}^{3}$} \\
\hline & $\begin{array}{c}\mathbf{L Y} \\
{[\mathrm{kPh} / \mathrm{MeV}]} \\
\end{array}$ & $\begin{array}{c}\text { CTR } \\
{[\mathrm{ps}]} \\
\end{array}$ & $\begin{array}{c}\mathbf{L Y} \\
{[\mathrm{kPh} / \mathrm{MeV}]} \\
\end{array}$ & $\begin{array}{c}\text { CTR } \\
{[\mathrm{ps}]} \\
\end{array}$ \\
\hline $\begin{array}{l}\text { LYSO } \\
\text { (CPI) }\end{array}$ & 17.4 & 184 & 13.7 & 192 \\
\hline $\begin{array}{c}\text { LYSO } \\
\text { (Proteus) }\end{array}$ & 18.0 & 175 & 13.9 & 188 \\
\hline $\begin{array}{c}\text { LYSO } \\
(\text { SIPAT) } \\
\end{array}$ & 19.0 & 206 & 12.0 & 223 \\
\hline
\end{tabular}

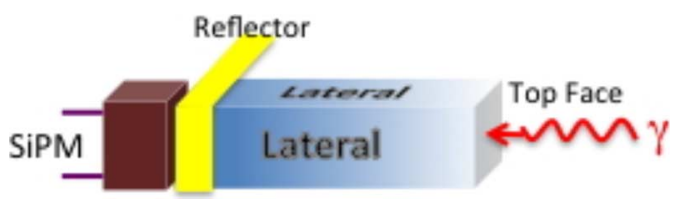

Fig. 9. Sketch of the crystal-photodetector geometry, showing the various crystal faces to be covered with a reflector or absorber.

in fair agreement with [26]. These losses are due to self-absorption, crystal impurities and surface flaws, but also imperfect reflectivity of the wrapping material. Thus, longer crystals, prone to a buildup of photon reflections, increase the probability of light transmission losses.

On the other hand, CTR improves between $13 \% / \mathrm{cm}$ and $19 \% / \mathrm{cm}$ for crystal lengths from $\sim 5$ to $20 \mathrm{~mm}$, also in good agreement with [26] and, by linear regression, levels off between 130 ps and 170 ps FWHM. Best result obtained in this work was a CTR of 140 ps FWHM with $4.6 \mathrm{~mm}$ long LSO:Ce:Ca-co-doped crystals.

\section{Comparison of Crystals With Different Cross Sections}

Similarly to the previous discussion this investigation involved a number of LY and CTR measurements that were made as a function of crystal cross section. Small section crystals, also called pixels, are favorable to provide the optimum in spatial resolution. We have tested this with LYSO crystals of same length $(10 \mathrm{~mm})$ but of two different sections from three different manufacturers. Table IV shows the results of these measurements.

We observe that despite a significant drop of $\sim 30 \%$ in LY the CTR is less impaired by the smaller crystal section. The corresponding increases in CTR range from 4\% (CPI crystal) to $7 \%$ (Proteus crystal) and $8 \%$ (SIPAT crystal).

\section{E. Effect of Crystal Wrapping on LY and CTR}

In the foregoing tests the crystals were conditioned to what is known to give the best results in terms of LY and CTR. This is commonly achieved with 'full' wrapping of the scintillator with a reflector, preferably with Teflon. To gain a more detailed insight into the effects of reflective material and light losses at the crystal faces, simulations, using LITRANI [33], and tests with different reflectors and wrapping methods were performed. As the illustration in Fig. 9 indicates, one either wraps the entire crystal, i.e., its top and four, lateral faces (full wrapping), or

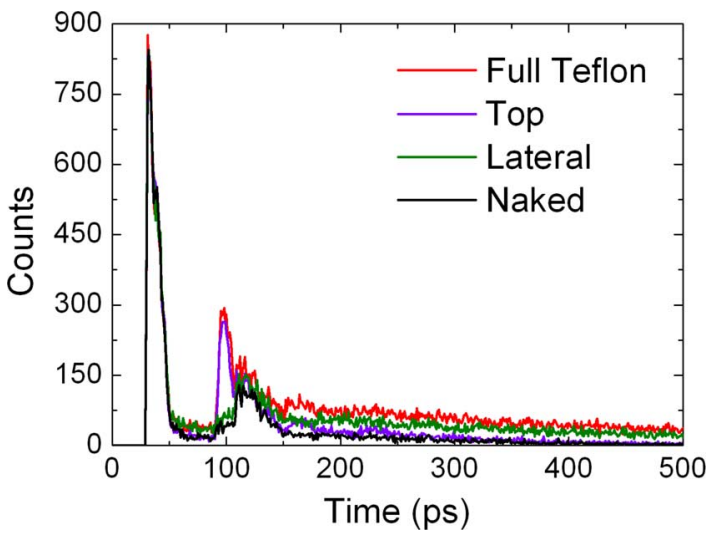

Fig. 10. Histogram of extracted photons in terms of time latency for four different reflector scenarios. 100'000 photons are generated in the middle of a $2 \times 2 \times 10 \mathrm{~mm}^{3}$ crystal. The simulations took into account a measured absorption length of $0.36 \mathrm{~m}[28]$ and $100 \mu \mathrm{m}$ diffusing edges ${ }^{5}$.

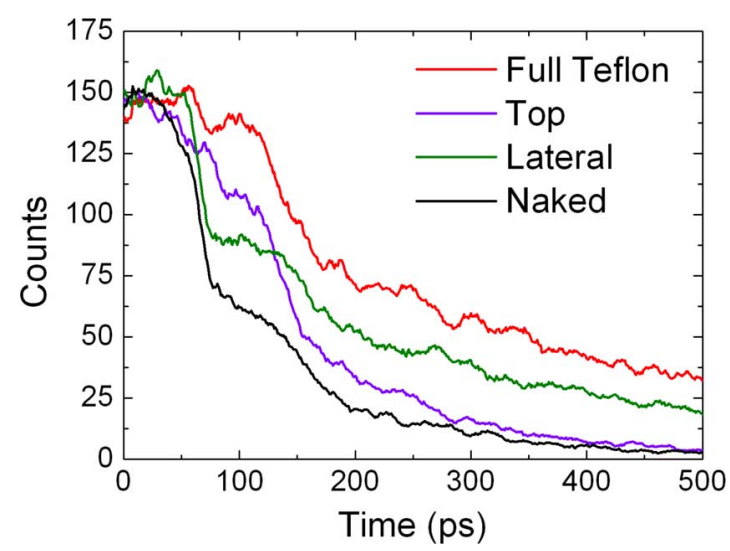

Fig. 11. Histogram of extracted photons in terms of time latency. Here 100'000 photons are generated uniformly in the entire crystal $\left(2 \times 2 \times 10 \mathrm{~mm}^{3}\right)$. The simulations took into account a measured photon absorption length of $0.36 \mathrm{~m}$ [28] and $100 \mu \mathrm{m}$ diffusing edges.

covers it only partially with a reflector or nearly absorbing black paper.

In Fig. 10 we show the latency of extracted photons [34], [35], from 100 '000 generated in the middle of a LYSO- $2 \times 2 \times 10$ $\mathrm{mm}^{3}$-crystal, under four different reflector scenarios, i.e., (i) with no reflector at all ('naked'), (ii) with only 'top' coverage with Teflon, (iii) 'lateral' wrapping of the crystal with Teflon and (iv) 'full' wrapping of the crystal.

In the case of releasing the generated photons from the center of the crystal (Fig. 10), about $9.5 \%$ of the extracted photons are encountered with very low latency $t<50$ ps, i.e., when no reflection took place. These are direct photons where the reflector plays no role. Reflected photons, however, with a latency of $\geq 100 \mathrm{ps}$ are clearly favored if full Teflon-wrapping (best) or partial wrapping at the top of the crystal is applied. The poorest photon yield results from an unwrapped (naked) or laterally only wrapped crystal for which the photon path is the longest. Fig. 11 illustrates this situation more visibly when the photons are, unlike before, generated and released uniformly in the crystal.

\footnotetext{
${ }^{5}$ The 'diffusing edge' is a $100 \mu \mathrm{m}$ thick zone at the edge of the crystal leading to diffusion of light when exiting the crystal.
} 
TABLE V

MeAsured Light Yield AND COINCIDENCE Time Resolution For Four WRAPPING SCENARIOS

\begin{tabular}{|c|c|c|c|c|}
\hline \multirow{2}{*}{ Wrapping } & \multirow{2}{*}{$\begin{array}{l}\text { Relative } \\
\mathrm{LY}^{*}[\%]\end{array}$} & \multicolumn{3}{|c|}{ CTR [ps] FWHM } \\
\hline & & $\begin{array}{c}\text { LSO } \\
\text { Ce:Ca }\end{array}$ & $\begin{array}{l}\text { LSO } \\
\text { CTI } \\
\end{array}$ & $\begin{array}{l}\text { LSO } \\
\text { PML } \\
\end{array}$ \\
\hline $\begin{array}{ll}\text { Top: } & \text { Teflon } \\
\text { Lateral: } & \text { Teflon }\end{array}$ & 100 & 170 & 190 & 206 \\
\hline $\begin{array}{l}\text { Top: } \quad \text { none } \\
\text { Lateral: } \text { Teflon }\end{array}$ & 77 & 195 & 224 & 237 \\
\hline $\begin{array}{l}\text { Top: Teflon } \\
\text { Lateral: none }\end{array}$ & 76 & 177 & 207 & 224 \\
\hline $\begin{array}{l}\text { Top: black } \\
\text { Lateral: } \text { black }\end{array}$ & 46 & 252 & 278 & 309 \\
\hline
\end{tabular}

${ }^{*} \mathrm{LY}$ is referenced and related to the light yield as achieved with Teflon wrapping on both the lateral and top faces $(100 \%)$. All crystals are $2 \times 2 \times 10 \mathrm{~mm}^{3}$. "Black" denotes wrapping with black tape.

TABLE VI

MEASUREMENTS OF LY AND CTR FOR FOUR DIFFERENT REFLECTORS

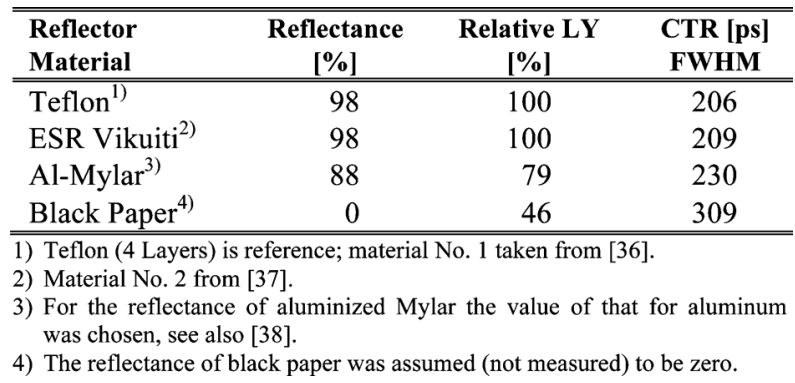

The non-point-like light emission leads, as expected, to a less pronounced appearance (smearing) of photons travelling towards the photodetector $(\mathrm{t}<100 \mathrm{ps})$ while it enhances the effect of the reflector boosting the number of photons according to the different propagation modes. The simulations therefore clearly demonstrate that proper wrapping increases the number of photons collected. On the other hand reflected photons, albeit copious, do not necessarily contribute to an improvement in time resolution as they arrive late following multiple reflections [19].

In Table $\mathrm{V}$ we summarize the LY and CTR measurements made with three $2 \times 2 \times 10 \mathrm{~mm}^{3}$-LSO-crystals in different wrapping scenarios.

These results show that, congruent with the outcome of our simulations of light extraction efficiency, full Teflon wrapping not only yields best performance in terms of LY but also in terms of CTR. In Table VI we summarize our results for four different reflector materials.

\section{SUMMARY AND CONCLUSION}

Our studies demonstrate that a CTR of well below 200 ps FWHM can be achieved with common crystals such as LSO and its derivatives LYSO and LGSO and a crystal length of $10 \mathrm{~mm}$. A new doping agent (calcium) applied together with cerium traces in LSO has produced very promising results with a CTR of 170 ps FWHM, also with $10 \mathrm{~mm}$ long crystals. This translates into a single detector resolution of better than $120 \mathrm{ps}$ FWHM.

Another important finding, as demonstrated by Table III, is that crystal quality in terms of LY for the same type crystal can vary between manufacturers by as much as $40 \%$. Nassalski et al. [39] have found that even between same crystal-specimen from the same manufacturer, the quality of crystals may not be uniform.

There is strong correlation between the crystal's LY and its potential time resolution, emphasizing once more the photostatistical impact on the temporal resolution. For the same emission decay time, higher LY always leads to higher time resolution.

Among the two tested crystals with light emission decay times well below 40 ns, i.e., LuAG:Pr $(20$ ns $)$ and LSO:Ce:Ca-co-doped (30 ns), only the latter could play this advantage as it emits, similar to the other LSO members, in the high PDE-region of the SiPM photodetector. LuAG:Ce, on the other hand, not 'handicapped' by unfavorable UV emission failed on terms of too long an emission decay time ( $60 \mathrm{~ns})$.

Encouraging results were achieved in view of the objectives set for the EndoTOFPET-US-project where small section crystals or 'fibers' of only $0.75 \times 0.75 \mathrm{~mm}^{2}$ section are to be employed. Despite a sizable drop in light output of $\sim 30 \%$ as compared to $2 \times 2 \mathrm{~mm}^{2}$-crystals, CTR remained basically unaffected, i.e., within $4 \%-8 \%$, and still below 200 ps FWHM.

Furthermore, with the appearance of the Hamamatsu $4 \times 4$ monolithic MPPC arrays, like the S11827-3344MG, scintillator pixels of $3 \times 3 \mathrm{~mm}^{2}$ can be grouped to matrices of crystals with a minimum of dead space between them. Such a scheme would ensure constructing compact detector modules for the EndoTOFPET-US system. The question of isolating the individual fibers in such a matrix and optimizing the light output of each still demands more studies on suitable reflectors and wrapping techniques. Our measurements nonetheless exemplify that so-called full wrapping and the use of Teflon or Vikuiti as the reflector indisputably lead to highest $\mathrm{LY}$, in agreement with our MC simulations. On the other hand, a CTR of $<200$ ps FWHM could also be achieved with partial wrapping, in particular of the top face of the crystals only. More work needs to be invested here, specifically to improve light transport from the scintillator to the photodetector and to enhance the direct photon collection [35].

Although of lesser importance, it should be emphasized that throughout this study relatively 'long' $(10 \mathrm{~mm})$ crystals were used, in an aim to balance temporal resolution, gamma detection efficiency and cost. Were it to aim at highest time resolution only, very short $(<4.5 \mathrm{~mm})$ LSO:Ce:Ca-co-doped crystals were shown to yield a CTR of 140 ps FWHM, the best result in our entire test series and fully in line with [40].

\section{ACKNOWLEDGMENT}

The authors wish to express their gratitude to D. Deyrail and A. Machard of the CERN PH-CMX Group who prepared the numerous crystals and special test facilities for the authors' measurements. Their devotion to the team and the success of this project is highly appreciated. 


\section{REFERENCES}

[1] EndoTOFPET-US:, "Novel multimodal endoscopic probes for simultaneous PET/ultrasound imaging for image-guided interventions," European Union 7th Framework Program (FP7/2007-2013) under Grant Agreement 256984 HEALTH-2010.1.2-1.

[2] J. S. Karp et al., "Benefit of time-of-flight in PET: Experimental and clinical results," J. Nucl. Med., vol. 49, no. 3, pp. 462-470, Mar. 2008.

[3] W. W. Moses, "Recent advances and future advances in time-of-flight PET," Nucl. Instrum. Methods Phys. Res. A, vol. 580, pp. 919-924, 2007.

[4] S. Surti et al., "Performance of Philips Gemini TF PET/CT scanner with special consideration for its time-of-flight imaging capabilities," J. Nucl. Med., vol. 48, no. 3, pp. 471-480, Mar. 2007.

[5] M. E. Casey, Improving PET With HD•PET + Time of Flight [Online]. Available: http://www.siemens.com/mi

[6] B. W. Jakoby et al., "Physical and clinical performance of the mCT time-of-flight PET/CT scanner," Phys. Med. Biol., vol. 56, pp. 2375-2389, 2011.

[7] H. Zaidi and A. Alavi, "Current trends in PET and combined (PET/ CTand PET/MR) systems design," PET Clin., vol. 2, pp. 109-123, 2007.

[8] S. Gundacker et al., "A systematic study to optimize crystal-SiPM photodetectors for highest time resolution," IEEE Trans. Nucl. Sci., vol. 59, no. 5, pp. 1798-1804, Oct. 2012.

[9] F. Anghinolfi et al., "NINO: An ultrafast low-power front-end amplifier discriminator for the time-of-flight detector in the ALICE experiment," IEEE Trans. Nucl. Sci., vol. 51, no. 5, pp. 1974-1978, Oct. 2004.

[10] P. Jarron et al., "Time based readout of a silicon photomultiplier (SiPM) for time of flight positron emission tomography (TOF-PET)," in Proc. 2009 IEEE Nuclear Science Symp. Conf. Rec., Jan. 2010, pp. 1212-1219, 2009.

[11] LeCroy DDA 735Zi (40GS/s).

[12] F. Powolny, "Characterization of time resolved photodetector systems for positron emission tomography," Ph.D. dissertation, Université de Neuchatel, Neuchatel, Switzerland, May 7, 2009.

[13] P. Lecoq et al., "Progress on photonic crystals," in Proc. IEEE Nuclear Science Symp. Conf. Rec., Oct. 2010, pp. 1970-1975.

[14] F. Powolny et al., "Time-based readout of a silicon photomultiplier (SiPM) for time of flight positron emission tomography (TOF-PET)," in Proc. IEEE Nuclear Science Symp. Conf. Rec., Oct. 2009, pp. $1212-1219$.

[15] P. Lecoq et al., "Factors influencing time resolution of scintillators and ways to improve them," in Proc. IEEE Nuclear Science Symp. Conf. Rec., Oct. 2009, pp. 1880-1885.

[16] D. Henseler et al., "SiPM performance in PET applications: An experimental and theoretical analysis," in Proc. IEEE Nuclear Science Symp. Conf. Rec., Oct. 24-Nov. 1 2009, pp. 1941-1948.

[17] M. G. Bisogni et al., "Characterization of Ca codoped LSO: Ce scintillators coupled to SiPM for PET applications," Nucl. Instrum. Methods Phys. Res. A, vol. 628, pp. 423-425, 2011.

[18] W. S. Choong, "The timing resolution of scintillation-detector systems: Monte Carlo analysis," Phys. Med. Biol., vol. 54, pp. 6495-6513, 2009.

[19] P. Lecoq, "New approaches to improve timing resolution in scintillators," in Proc. SCINT 2011, Giessen, Germany.

[20] R. F. Post and L. I. Schiff, "Statistical limitations on the resolving time of a scintillation counter," Phys. Rev., vol. 80, pp. 1113-1113, 1950.
[21] F. J. Lynch, "Improved timing with NaI(Tl)," IEEE Trans. Nucl. Sci., vol. NS-13, no. 3, pp. 140-147, Jun. 1966.

[22] Y. Shao, "A new timing model for calculating the intrinsic timing resolution of a scintillator detector," Phys. Med. Biol., vol. 52, pp. 1103-1117, 2007.

[23] S. Seifert, H. T. van Dam, and D. R. Schaart, "The lower bound on the timing resolution of scintillation detectors," Phys. Med. Biol., vol. 57, pp. 1797-1814, 2012.

[24] M. W. Fishburn and E. Charbon, "System tradeoffs in gamma-ray detection utilizing SPAD arrays and scintillators," IEEE Trans. Nucl. Sci., vol. 57, no. 5, pp. 2549-2557, Oct. 2010.

[25] S. E. Derenzo, M. J. Weber, W. W. Moses, and C. Dujardin, "Measurements of the intrinsic rise times of common inorganic scintillators," IEEE Trans. Nucl. Sci., vol. 47, no. 3, pp. 860-864, Jun. 2000.

[26] C. L. Kim et al., "Dependence of timing resolution on crystal size for TOF PET," in Proc. IEEE Nuclear Science Symp. Conf. Rec., Oct./Nov. 2007, vol. 4.

[27] M. Kronberger et al., "Determination of the absolute light yields of LuYAP and LYSO," in Proc. IEEE Nuclear Science Symp. Conf. Rec., Oct. 2008, pp. 1153-1157.

[28] M. Kronberger, "Optimization of the light extraction from heavy inorganic scintillators," Ph.D. dissertation, Univ. Technology, Vienna, Austria, 2008.

[29] U. Heinrichs et al., "Statistical studies on the light output and energy resolution of small LSO single crystals with different surface treatments combined with various reflector materials," Nucl. Instrum. Methods Phys. Res. A, vol. 486, pp. 60-66, 2002.

[30] S. Scheu et al., "Studies on wrapping materials and light collection geometries in plastic scintillators," Nucl. Instrum. Methods Phys. Res. $A$, vol. 567, pp. 345-349, 2006.

[31] M. A. Spurrier et al., "Effects of $\mathrm{Ca}^{2+}$ Co-doping on the scintillation properties of LSO:Ce," IEEE Trans. Nucl. Sci., vol. 55, no. 3, pp. 1178-1182, Jun. 2008.

[32] P. Eckert et al., "Characterization studies of silicon photomultipliers," Nucl. Instrum. Methods Phys. Res. A, vol. 620, pp. 217-226, 2010.

[33] F.-X. Gentit, "Litrani: A general purpose Monte-Carlo program simulating light propagation in isotropic or anisotropic media," Nucl. Instrum. Methods Phys. Res. A, vol. 486, no. 1-2, pp. 35-39, 2002.

[34] K. Pauwels et al., "Effect of aspect ratio on the light output of scintillators," IEEE Trans. Nucl. Sci., vol. 59, no. 5, pp. 2340-2345, Oct. 2012.

[35] A. Knapitsch et al., "Effects of photonic crystals on the light output of heavy inorganic scintillators," in Proc. Nuclear Science Symp. and Medical Imaging Conf., Oct. 2011.

[36] M. Janecek and W. Moses, "Optical reflectance measurements for commonly used reflectors," Lawrence Berkeley National Laboratory, Jun. 17, 2009 [Online]. Available: http://escholarship.org/uc/item/82r9k9rn

[37] 3M, Vikuiti Enhanced Specular Reflector (ESR) [Online]. Available: http://multimedia.3m.com $/ \mathrm{mws} /$ mediawebserver?mwsId $=66666$ UuZjcFSLXTtMxMyOXM6EVuQEcuZgVs6EVs6E666666- -\&fn $=$ ESR $\% 20$ ss 2 .pdf

[38] J. Hanlon, Handbook of Package Engineering, 1st ed. Lancaster, PA, USA: Technomic Publishing, 1992, ISBN 0-87762-924-2, Chapter 3, Films and Foils.

[39] A. Nassalski et al., "Comparative study of scintillators for PET/CT detectors," IEEE Trans. Nucl. Sci., vol. 54, no. 1, pp. 3-10, Feb. 2007.

[40] S. Seifert et al., "A novel timing model for SiPM-Based scintillation detectors: Theory and experimental validation," IEEE Trans. Nucl. Sci., vol. 59, no. 1, pp. 190-204, Feb. 2012. 